\title{
Photocurrent Polarization Anisotropy of Randomly Oriented Nanowire Networks
}

\author{
Yanghai Yu, ${ }^{\dagger}$ Vladimir Protasenko, $\ddagger^{\ddagger}$ Debdeep Jena, ${ }^{\S}$ Huili (Grace) Xing,,$\$$ and \\ Masaru Kuno
}

\author{
Department of Chemical and Biomolecular Engineering, Department of Chemistry and \\ Biochemistry and Notre Dame Radiation Laboratory, Department of Electrical \\ Engineering, University of Notre Dame, Notre Dame, Indiana 46556
}

Received January 4, 2008; Revised Manuscript Received March 5, 2008

\begin{abstract}
While the polarization sensitivity of single or aligned NW ensembles is well-known, this article reports on the existence of residual photocurrent polarization sensitivities in random NW networks. In these studies, CdSe and CdTe NWs were deposited onto glass substrates and contacted with Au electrodes separated by 30-110 $\mu \mathrm{m}$ gaps. SEM and AFM images of resulting devices show isotropically distributed NWs between the electrodes. Complementary high resolution TEM micrographs reveal component NWs to be highly crystalline with diameters between 10 and $20 \mathrm{~nm}$ and with lengths ranging from 1 to $10 \mu \mathrm{m}$. When illuminated with visible (linearly polarized) light, such random NW networks exhibit significant photocurrent anisotropies $\rho=0.25(\sigma=0.04)[\rho=0.22(\sigma=0.04)]$ for CdSe (CdTe) NWs. Corresponding bandwidth measurements yield device polarization sensitivities up to $100 \mathrm{~Hz}$. Additional studies have investigated the effects of varying the electrode potential, gap width, and spatial excitation profile. These experiments suggest electrode orientation as the determining factor behind the polarization sensitivity of NW devices. A simple geometric model has been developed to qualitatively explain the phenomenon. The main conclusion from these studies, however, is that polarization sensitive devices can be made from random NW networks without the need to align component wires.
\end{abstract}

Introduction. Semiconductor nanowires (NWs) are onedimensional (1D) nanostructures that exhibit unique optical and electrical properties, including strong polarization sensitivities, ${ }^{1}$ low lasing thresholds, ${ }^{2-4}$ enhanced carrier mobilities, ${ }^{5,6}$ and potential ballistic transport capabilities. ${ }^{7}$ Of these features, the intrinsic polarization anisotropy $(\rho)$ of NWs has attracted significant attention due to the potential for creating polarization sensitive devices. ${ }^{8-15}$ In this regard, emission $\rho$-values of $\sim 0.6$ have been observed in lithographically defined quantum wires. ${ }^{8}$ Much larger excitation/emission $\rho$-values of $\sim 0.91$ have been reported in free-standing InP NWs. ${ }^{1}$ Individual solution grown CdSe NWs also exhibit large absorption (emission) polarization anisotropies of $\rho \approx$ $0.77(\rho \approx 0.76)$. At the ensemble level, smaller absorption (emission) anisotropy values of $\rho \approx 0.24(\rho \approx 0.27$ ) have been observed and stem from the incomplete alignment of wires in dielectrophoretically aligned ensembles. ${ }^{11}$

In all cases, polarization anisotropies have been determined using the expression $\rho=\left(I_{\|}-I_{\perp}\right) /\left(I_{\|}+I_{\perp}\right)$, where $I_{\|}$and $I_{\perp}$ are the intensities of the incident (or emitted) light polarized parallel versus perpendicular to the NW growth axis. In

\footnotetext{
* Corresponding author. E-mail: mkuno@nd.edu.

† Department of Chemical and Biomolecular Engineering, University of Notre Dame.

$¥$ Department of Chemistry and Biochemistry and Notre Dame Radiation Laborator, University of Notre Damey.

$\S$ Department of Electrical Engineering, University of Notre Dame.
}

complementary photocurrent measurements, $I_{\|}$and $I_{\perp}$ are the magnitudes of the photogenerated current with the incident excitation polarized parallel versus perpendicular to the NW axis.

In studying the photoconductivity of aligned NWs, ${ }^{16}$ we have found that photocurrent anisotropies can be obtained from random NW networks. Although initially a surprise, such polarization sensitivities have previously been predicted by Ruda and Shik for the emission originating from NW ensembles in solution. This has since been experimentally observed in solutions of randomly oriented CdSe nanorods (NRs), where the emission appears polarized under polarized excitation. ${ }^{17}$ The current study further confirms the underlying idea behind the existence of residual polarization anisotropies in random NW ensembles within the context of photocurrent measurements.

In the former study by Ruda and co-workers, a residual emission polarization has been explained by noting that the incident polarized light preferentially excites a subset of NWs within the ensemble. This occurs in those wires oriented near parallel to the incident excitation polarization. Such NWs are most sensitive to the light due to absorption anisotropies arising from either dielectric contrast effects or intrinsic absorption selection rules. ${ }^{18}$ Subsequent (linearly polarized) light is then emitted from these wires. The net result is a suppression of randomness in the system, enabling the observation of a measurable emission anisotropy. The extent 


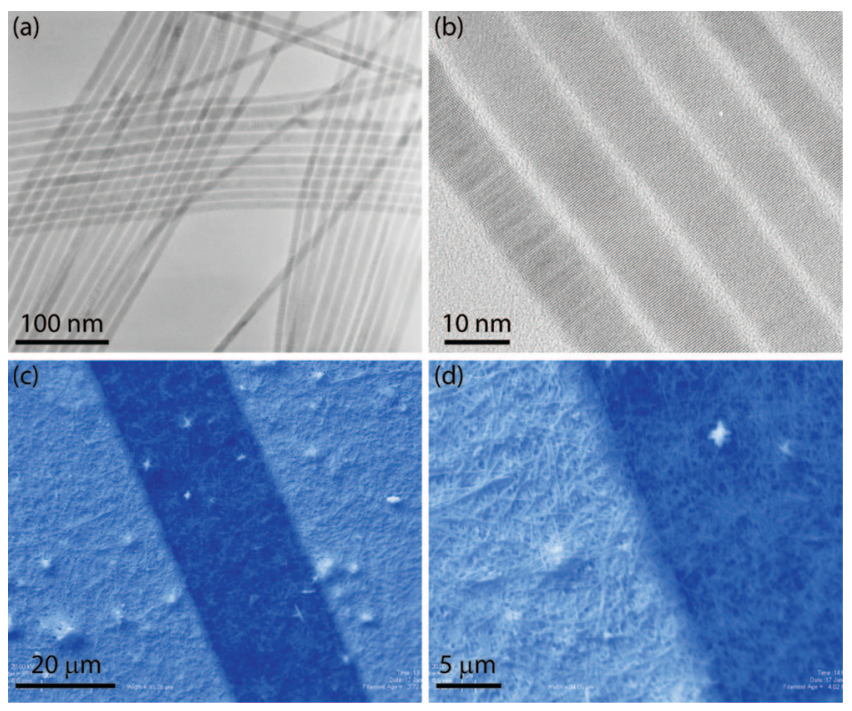

Figure 1. (a) Low and (b) high resolution TEM micrographs of straight CdSe NWs. (c) Low and (d) high magnification SEM micrographs of the electrode/channel junction. In either case, the dark region is the gap containing randomly distributed NWs. Adjacent lighter colored regions represent evaporated gold electrodes over the wires.

of this polarization "memory" depends on how quickly individual wires in solution tumble or reorient, erasing any excitation/emission spatial correlations.

By the same token, static NW networks exhibit similar random distributions. However, in the current experiment, an external dc electric field imparts directionality to the system by favoring the dissociation of $1 \mathrm{D}$ excitons in wires oriented primarily along the electric field lines. Resulting photocurrent anisotropies can, in turn, be sampled by photocurrent measurements and constitute the scope of this paper. A rudimentary geometric model has been developed to qualitatively explain trends observed in the data. The most important result from this study, however, is the conclusion that polarization sensitive devices can be made from randomly oriented NWs without having to align them.

Experimental Section. CdSe and CdTe NWs were synthesized by following a recently developed solution - liquidsolution (SLS) growth scheme (Figure 1a). ${ }^{19-21}$ Briefly, for CdSe NWs, $\sim 1.5 \mathrm{~nm}$ diameter $\mathrm{Au} / \mathrm{Bi} \mathrm{NPs}^{19}$ were used to catalyze the nucleation and growth of NWs in a reaction medium consisting of trioctylphosphine oxide (TOPO), trioctylphosphine selenide (TOPSe), cadmium oxide, and octanoic acid. High-resolution TEM images of resulting wires (Figure 1b) reveal them to be crystalline, with mean diameters ranging from 10 to $20 \mathrm{~nm}$ (Additional micrographs, Supporting Information). Corresponding ensemble diameter distributions range from 25 to $30 \%$ with significantly smaller 3-6\% intrawire variations. Similar values are obtained for CdTe NWs. ${ }^{21}$ The NW length in both cases exceeds $1 \mu \mathrm{m}$, as evident in low resolution TEM and complementary atomic force microscope (AFM) measurements.

Samples for photocurrent measurements were prepared in two steps. NW suspensions were first dropcast onto glass coverslips. Back of the envelope calculations using TEM sizing yield estimated surface densities between $10^{9}$ to $10^{10}$ $\mathrm{NWs} / \mathrm{cm}^{2}$. A thin $(30,90$, or $110 \mu \mathrm{m}$ diameter) gold wire was then taped over the NW film to act as a shadow mask for the subsequent evaporation of gold contacts. Approximately $40 \mathrm{~nm}$ of Au was deposited over the wires to create electrodes whereupon the mask was removed to complete the device (device schematic, Supporting Information). Parts $\mathrm{c}$ and $\mathrm{d}$ of Figure 1 show SEM micrographs of the resulting assembly. Of note is the apparent random NW distribution within the gap.

For photocurrent measurements, samples were placed on top of a piezo stage attached to a home-built inverted optical microscope. A $532 \mathrm{~nm}(2.33 \mathrm{eV})$ green laser diode was used to excite the sample. Typical laser spot sizes (fwhm) ranged from 16 to $20 \mu \mathrm{m}$ unless otherwise stated. Corresponding excitation intensities varied from 3 to $5 \mathrm{~kW} / \mathrm{cm}^{2}$. Excitation spot diameters were controlled using a $+400 \mathrm{~mm}$ lens placed prior to the objective's back aperture. A sheet polarizer (100:1 quality factor) was mounted on a computer controlled stepper motor to enable angular control of the incident light polarization. Photocurrents as well as laser intensities were measured at each orientation of the polarizer using a homebuilt probe station coupled to a picoammeter (Kiethley) and an optical power meter (Newport). To correct for excitation intensity fluctuations, photocurrents were subsequently normalized to the laser power. Spectral sensitivities of the polarization anisotropy as well as complementary photocurrent action spectra between 480 and $670 \mathrm{~nm}(2.58-1.85 \mathrm{eV})$ were acquired with the tunable (grating dispersed) wavelength output from a supercontinuum white light source (Fianium).

Polarization anisotropy bandwidth measurements were conducted using a ferroelectric liquid crystal polarization rotator (Displaytech). The input signal from a function generator was varied between 0.5 to $2 \mathrm{kHz}$ to rotate the output linear polarization by $90^{\circ}$ at the prescribed frequency. Measured photocurrents were then fed into a preamplifier connected to a lock-in amplifier (SRS). At frequencies below $1 \mathrm{~Hz}$, photocurrents were measured directly with the picoammeter.

To address the possibility of nonuniform electric fields across the NW channel (resulting from local charge accumulation), several control experiments were conducted. AFM surface potential measurements were carried out with a Digital Instruments Nanoscope IV on samples used in above optical experiments. One of the electrodes was grounded while $+9.5 \mathrm{~V}$ was applied to the other $(E \approx 1.1$ $\times 10^{3} \mathrm{~V} / \mathrm{cm}$ ). In a second experiment, random NW networks were excited using variable width laser excitation spots. Rectangular slits with fixed height but with variable widths were inserted prior to the objective to shape the imaged spot on the sample. Resulting (rectangular) spot sizes ranged from $0.75 \mu \mathrm{m} \times 20 \mu \mathrm{m}$ to $12 \mu \mathrm{m} \times 20 \mu \mathrm{m}$. In the absence of charge accumulation, a linear potential drop across the gap and a corresponding photocurrent linear dependence with slit width is expected. 
Results and Discussion. Photocurrent Anisotropy of Randomly Oriented CdSe and CdTe NW Networks. At first glance, no polarization sensitivity is expected from randomly oriented NW ensembles. However, more rigorous modeling ${ }^{12}$ as well as recent emission polarization measurements on CdSe NR solutions ${ }^{17}$ reveal the existence of a polarization "memory" in isotropic NW or NR samples. In the current study, analogous polarization sensitivities have been measured in photocurrents obtained from static arrays of randomly oriented CdSe and CdTe NWs. Parts a and b of Figure 2 illustrate this by plotting the measured normalized photocurrent (dark current subtracted, representative $I-V$ curve, Supporting Information) as a function of excitation polarization angle (red triangles) for CdSe NWs (CdTe data, Supporting Information). Clear photocurrent oscillations with a $180^{\circ}$ periodicity are seen as the incident excitation polarization angle is rotated. Fits to a $\cos ^{2} \theta$ function (dashed line) reveal a substantial polarization anisotropy of $\rho \approx 0.28$. When averaged over 5 samples and 60 random excitation positions for a given system, obtained $\rho$-values are $\rho=0.25$ $(\sigma=0.04)[\rho=0.22(\sigma=0.04)]$ for CdSe $(\mathrm{CdTe})$ NWs. No apparent gap (or bias) dependencies of $\rho$ are seen in any of the samples.

To verify the origin of observed photocurrents and associated anisotropies, photocurrent action spectra of random NW devices were measured for both $\mathrm{CdSe}$ and $\mathrm{CdTe}$ wires. Resulting spectra were then compared to their complementary solution UV/visible extinction spectra (Figure $2 \mathrm{c}$, dashed line, $\mathrm{CdSe}$ ). The good agreement between the two verifies that measured photocurrents indeed originate from the NWs (CdTe NWs, Supporting Information).

To rule out any artifacts due to the imperfect polarization of the excitation (initially 100:1, deteriorating to $\sim 40: 1$ after reflecting off an $\mathrm{Al}$ mirror), identical anisotropy experiments were conducted using a sample consisting of a commercial, polarization insensitive, photodiode. Apart from illustrating imperfections in the incident laser polarization ( $\rho_{\text {appar }}$ ent $\sim 0.03$ ), parts a and $b$ of Figure 2 (blue circles) show that such contributions account for no more than $\sim 15 \%$ of observed $\rho$-values in actual NW network anisotropy measurements. Given this, the existence of a residual polarization anisotropy is confirmed.

What remains to be determined then is the origin of the directionality in the system. Figure $2 \mathrm{a}$ demonstrates that the photocurrent is sensitive to the orientation between the incident light polarization $\left(\vec{E}_{0}\right)$ and the external dc electric field $\left(\vec{E}_{\mathrm{dc}}\right)$. Specifically, the maximum photocurrent occurs when the light polarization is parallel to $\vec{E}_{\mathrm{dc}}$. Conversely, a minimum occurs when the incident light polarization is normal to $\vec{E}_{\mathrm{dc}}$. This strongly suggests that photocurrent anisotropies are greatly influenced by the orientation of the externally applied dc field.

To rule out the possibility that such residual anisotropies occur due to the local orientation of wires in a NW network, the following were investigated. First, the excitation spot was laterally modulated $\sim 20 \mu \mathrm{m}$ in the vertical direction (parallel to the Au electrodes) using a sinusoidal $50-100 \mathrm{~Hz}$ voltage applied to the sample
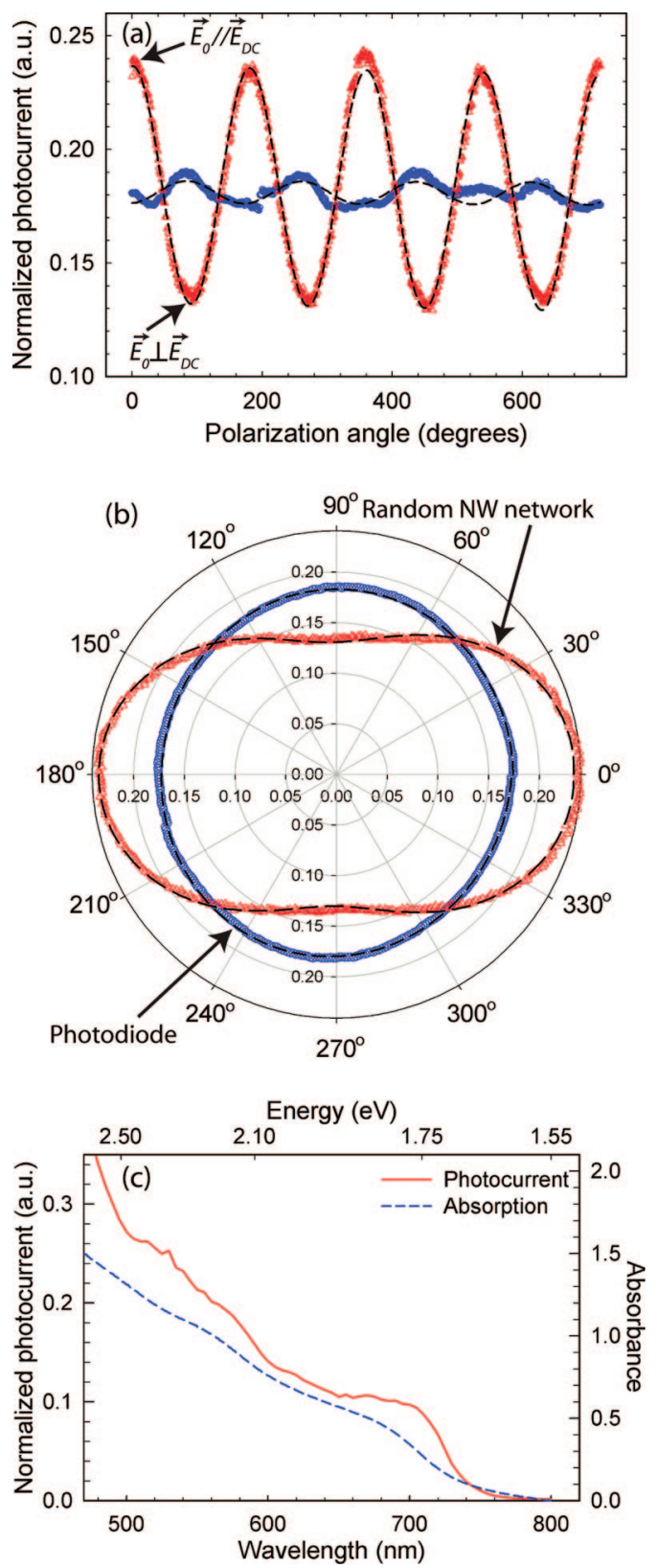

Figure 2. (a) Photocurrent anisotropy of a random CdSe NW network (red triangles). Corresponding control using a polarization insensitive photodiode (blue circles). Maximum (minimum) photocurrents are detected when the incident light polarization $\left(\vec{E}_{0}\right)$ is parallel (perpendicular) to the external dc electric field $\left(\vec{E}_{\mathrm{dc}}\right)$ orientation. Dashed lines are $\cos ^{2} \theta$ fits to the data. (b) Associated polar plot. (c) Linear absorption and associated photocurrent action spectrum of a CdSe NW network.

piezostage. This ensures that measured photocurrents are insensitive to local alignment effects. In a complementary experiment, the diameter of the excitation spot was varied between 10 and $40 \mu \mathrm{m}$ to average the photocurrent over wider regions of the channel (Supporting Information). In both cases, no significant differences in measured $\rho$-values were observed. These results suggest that NW films do not possess local directionality, hinting at a deeper origin for the residual anisotropy. Corroborating this, local alignment effects should alter the phase angle of the photocurrent 

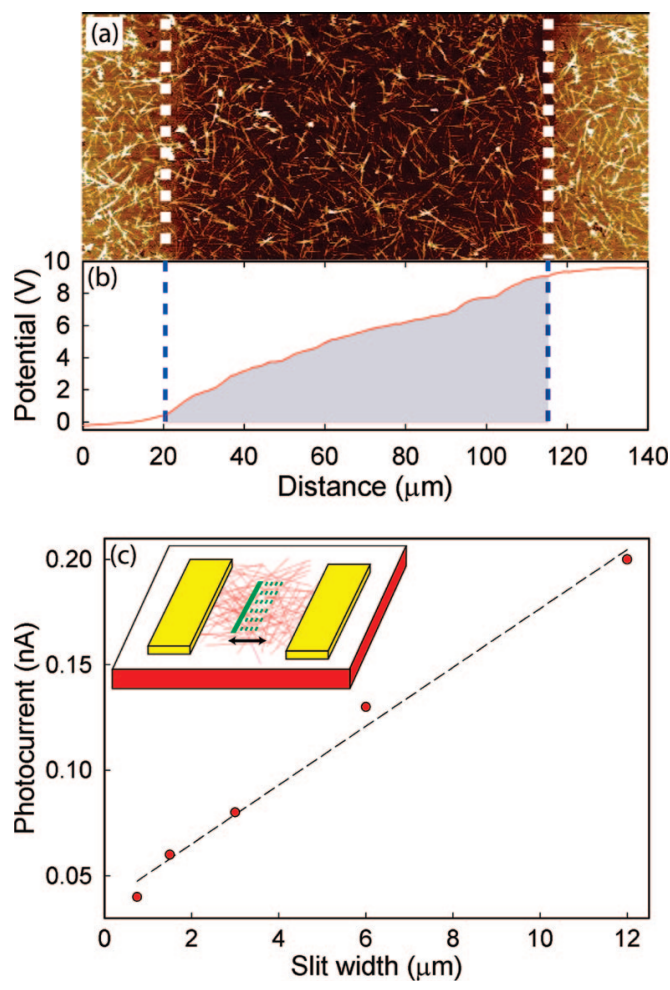

Figure 3. (a) AFM topography of NWs in the gap. (b) Corresponding surface potential measured across the gap $(9.5 \mathrm{~V}, E \approx 1.1 \times$ $10^{3} \mathrm{~V} / \mathrm{cm}$ ). (c) Changes in photocurrent with excitation slit width (constant height, $20 \mu \mathrm{m}$ ).

anisotropy trajectory because the amount of absorbed light and hence the number of photogenerated carriers is determined by the mutual orientation between the light polarization and the NW axis. However, this was not observed when the excitation spot was translated sequentially across the gap (Supporting Information).

An additional concern is that the localization of photogenerated carriers trapped at interfaces or on NW surfaces could affect the externally applied electric field through local fields having unpredictable directionalities. This would then alter the magnitude of measured (global) $\rho$-values. To address this, two additional control experiments were conducted.

The first entails AFM-based surface potential measurements across the NW channel. Figure 3 illustrates the AFM topography (Figure 3a) and the corresponding surface potential (Figure $3 b$ ) measured across the gap. In the latter data, the potential increases almost linearly from the negative to the positive electrode. This shows that a spatially uniform external field exists within the random NW network under an applied bias.

However, to better investigate the uniformity of the potential under more realistic conditions (i.e., under illumination) experiments employing variable sized excitation spots were conducted. In particular, the width of a rectangular excitation spot, aligned with the long axis parallel to the electrodes, was varied (Figure 3c, inset). If charge accumulation occurs at NW interfaces due to the existence of longlived electron/hole traps, inhomogeneities in the applied field strength and direction should occur within the excitation region. By altering the width of the spot, larger/smaller numbers of traps are sampled. One therefore expects a weak dependence between the measured photocurrent and the excitation width because of the randomizing effect of competing local field anisotropies. However, the experimental data instead shows a pronounced linear dependence (Figure $3 c$ ). This suggests that effects of local electric fields associated with trapped charges are negligible compared to the directionality imposed by the external do field.

Both frequency and spectral bandwidth issues were subsequently investigated. In the former case, a ferroelectric liquid crystal polarization rotator was inserted into the optical train prior to the sample. Applying a periodic stepwise potential induces a $90^{\circ}$ rotation of the incident light polarization. Polarization switching frequencies between 0.5 and 2 $\mathrm{kHz}$ were applied. Obtained results (Supporting Information) show NW device bandwidths of approximately $100 \mathrm{~Hz}$ with measured (low frequency) $\rho$-values of $\rho \approx 0.23$, dropping to $\rho \approx 0.09$ at $100 \mathrm{~Hz}$. This behavior may be explained by the slow mobility of free carriers in the film, stemming from hopping transport within the random NW network. Estimated carrier mobilities are $\mu_{\mathrm{e}(\mathrm{h})}^{\mathrm{film}} \sim 10^{-4} \mathrm{~cm}^{2} / \mathrm{Vs}$.

Spectral bandwidth measurements were conducted at wavelengths ranging from 480 to $670 \mathrm{~nm}(2.58-1.85 \mathrm{eV})$. Nearly uniform $\rho$-values $(\rho \approx 0.26)$ were found across the entire spectral range with a minimum (maximum) value of $\rho \approx 0.23(\rho \approx 0.28)$ at $532 \mathrm{~nm}(670 \mathrm{~nm})$ for CdSe NWs (Supporting Information). Both show no significant $\rho$ excitation wavelength dependencies in line with the expected behavior of thick NWs experiencing dielectric contrast effects.

Rudimentary Model for the Photocurrent Anisotropy. To estimate the number of photogenerated carriers created within the NW network, the magnitude of the incident light's electric field within a given wire was estimated classically. ${ }^{22}$ In this regard, nanowires are known to exhibit strong polarization sensitivities. ${ }^{1,11}$ However, several origins for this anisotropy exist. For thin NWs, with diameters below the corresponding bulk exciton Bohr radius, the energy spectrum and associated selection rules for transitions likely exhibit modifications due to confinement effects. ${ }^{13}$ For thicker wires, however, such polarization sensitivities can be explained classically. ${ }^{22}$ The latter approach is used here because NW diameters exceed twice the bulk exciton Bohr radius of either CdSe $\left(a_{\mathrm{B}}=5.6 \mathrm{~nm}\right)$ or CdTe $\left(a_{\mathrm{B}}=7.5 \mathrm{~nm}\right)$.

Obtained solutions contain a parallel component of the internal field, $E_{\|}$, with a magnitude identical to the incident external field, $E_{0 \mid l}$. A perpendicular component, $E_{\perp}$, with a magnitude largely attenuated $\left(E_{\perp}=\left\{2 \varepsilon_{\mathrm{m}} /\left(\varepsilon+\varepsilon_{\mathrm{m}}\right)\right\} E_{0 \perp}\right)$ also exists. In the last expression, $\epsilon\left(\epsilon_{\mathrm{m}}\right)$ is the dielectric constant of the NW (surrounding medium). For CdSe and CdTe, the bulk high frequency dielectric constant at the excitation wavelength $(\lambda=532 \mathrm{~nm}, 2.33 \mathrm{eV})$ is $\epsilon_{\mathrm{CdSe}}=7.21+2.61 i$ and $\epsilon_{\mathrm{CdTe}}=8.58+1.77 i{ }^{23}$ While the corresponding environment's dielectric constant is, in principle, that of air, NWs are placed at the air/glass interface when deposited onto a glass microscope coverslip. As a consequence, both the 

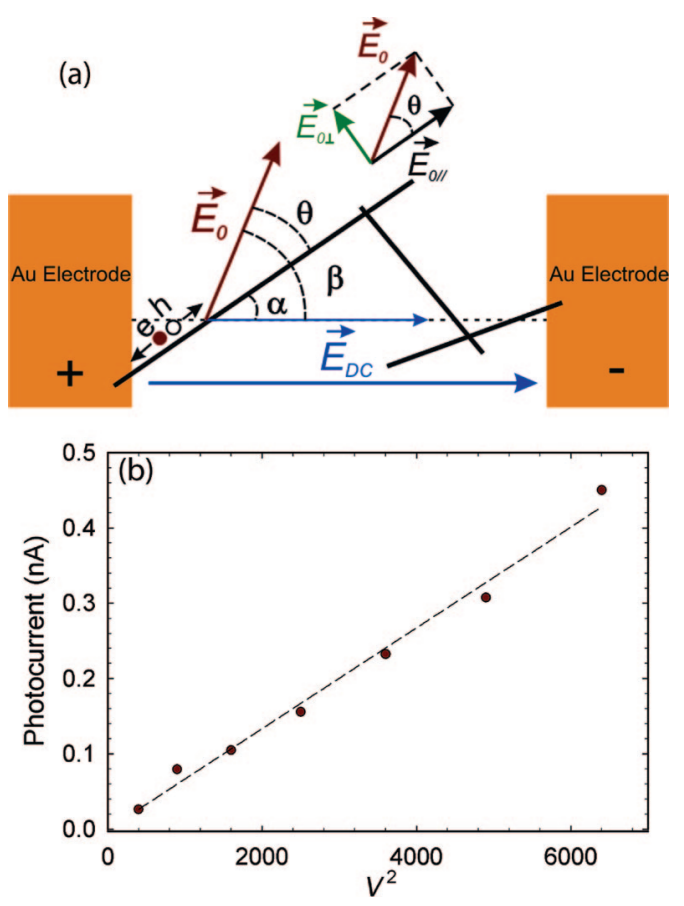

Figure 4. (a) Cartoon schematic of model considerations for calculating the residual photocurrent anisotropy. In the diagram, $\alpha$ $(\beta)$ represents the NW (incident polarization) orientation relative to the external dc field and $\theta$ is the angle between the light's polarization and the NW growth axis. $E_{0}$ is the magnitude of the incident light electric field. Likewise, $E_{\mathrm{dc}}$ is the magnitude of the applied dc electric field. (b) Effect of applied bias on the photocurrent.

dielectric constants of glass $\left(\epsilon_{\text {glass }}=2.3\right)$ and air $\left(\epsilon_{\text {air }}=1.0\right)$ were averaged to obtain $\epsilon_{\mathrm{m}}=1.65$.

The light intensity, $P$, within the NW was then found by squaring the internal electric field $\left(P \propto E^{2}=E_{\|}^{2}+E_{\perp}^{2}\right)$. Using our previous expressions for $E_{\|}$and $E_{\perp}$, we find

$$
P \propto E_{0}^{2}\left|\frac{2 \varepsilon_{\mathrm{m}}}{\varepsilon+\varepsilon_{\mathrm{m}}}\right|^{2} \sin ^{2}(\beta-\alpha)+E_{0}^{2} \cos ^{2}(\beta-\alpha)
$$

where $\alpha$ denotes the angle between the NW growth axis and the external dc field. Likewise $\beta$ is the angle between the incident light polarization vector and the external dc field (Figure 4a). The difference between these angles, $\beta-\alpha$, yields the angle, $\theta$, between the NW growth axis and the excitation polarization. Together with the NW absorption cross section, ${ }^{24}$ an estimate for the number of carriers generated within a NW at a given orientation relative to the incident light polarization is possible. This, however, does not account for the overall polarization sensitivity of a random NW network because an ensemble of randomly oriented wires is being excited in tandem.

We have previously shown (Figure 2a) that the origin of the random NW network polarization sensitivity is related to the relative orientation of the incident light polarization and the direction of the external dc field. In this regard, the role of the dc field in creating a residual anisotropy is thought to arise from changing the dissociation rate of strongly bound $(\sim 100-300 \mathrm{meV}) 1 \mathrm{D}$ excitons ${ }^{25}$ present in NWs. The effect is most prominent in wires closely aligned with the external dc field because its projection is largest within these wires. 1D excitons arise from the large permittivity difference between NWs and their surrounding medium ${ }^{26}$ and have recently been observed in carbon nanotubes ${ }^{27}$ as well as in CdSe NWs. ${ }^{28}$

To estimate the number of free carriers $\left(n_{\mathrm{e}(\mathrm{h})}\right)$ contributing to the observed photocurrent for wires at a give orientation relative to the external dc field, the magnitude of the dc field inside the NW was evaluated. This was done using identical considerations employed in deriving eq 1 . Specifically, the parallel component of the external dc field inside a given wire is written as $E_{\mathrm{dc}}|\cos \alpha|$ while the normal component is $\left\{2 \varepsilon_{\mathrm{m}} /\left(\varepsilon+\varepsilon_{\mathrm{m}}\right)\right\} E_{\mathrm{dc}}|\sin \alpha|$. The number of electrons and holes, $n_{\mathrm{e}(\mathrm{h})}$, contributing to the measured current is then assumed to be proportional to the external dc field strength through

$$
n_{e(h)} \propto E_{\mathrm{dc}}\left(|\cos \alpha|+\left|\frac{2 \varepsilon_{\mathrm{m}}}{\varepsilon+\varepsilon_{\mathrm{m}}} \sin \alpha\right|\right)
$$

Once separated, mobile electrons/holes travel through the NW network toward their respective electrodes. Because estimated NW densities $\left(10^{9}\right.$ to $10^{10} \mathrm{NWs} / \mathrm{cm}^{2}$, Experimental Section) are well above the percolation threshold $\left(1 /\langle L\rangle^{2} \sim\right.$ $4 \times 10^{6} \mathrm{NWs} / \mathrm{cm}^{2},{ }^{29}\langle L\rangle=5 \mu \mathrm{m}$ is the average NW length), each NW connects to many other wires with different orientations. Coupled to the fact that the electrode gap is larger than $\langle L\rangle$, carriers therefore likely undergo hopping transport to the electrodes. Corroborating this, estimated NW network mobilities are $\mu_{\mathrm{e}(\mathrm{h})}^{\text {film }} \sim 10^{-4} \mathrm{~cm}^{2} /(\mathrm{Vs})$ are easily $4-6$ orders of magnitude smaller than representative intraband mobilities $150-650 \mathrm{~cm}^{2} /(\mathrm{Vs})$ measured in other NWs. ${ }^{30}$

Carrier transport through this resistive NW network is thus modeled using the following effective carrier velocity

$$
v_{\mathrm{e}(\mathrm{h})} \propto \mu_{\mathrm{e}(\mathrm{h})}^{\mathrm{film}} E_{\mathrm{dc}}
$$

Because the current density, $j$, is proportional to the product of $n_{\mathrm{e}(\mathrm{h})}$ and $v_{\mathrm{e}(\mathrm{h})}$, eqs 2 and 3 then yield

$$
j \propto E_{\mathrm{dc}}^{2}\left(|\cos \alpha|+\left|\frac{2 \varepsilon_{\mathrm{m}}}{\varepsilon+\varepsilon_{\mathrm{m}}} \sin \alpha\right|\right)
$$

which, when combined with eq 1 , provides an expression for the overall photocurrent, $I$

$$
\begin{array}{r}
I \propto E_{0}^{2} E_{\mathrm{dc}}^{2}\left\{\left|\frac{2 \varepsilon_{0}}{\varepsilon+\varepsilon_{0}}\right|^{2} \sin ^{2}(\beta-\alpha)+\cos ^{2}(\beta-\alpha)\right\}\{|\cos \alpha|+ \\
\left.\left|\frac{2 \varepsilon_{\mathrm{m}}}{\varepsilon+\varepsilon_{\mathrm{m}}} \sin \alpha\right|\right\}
\end{array}
$$

If we now assume that the separation of carriers inside a $\mathrm{NW}$ is dominated primarily by the electric field component parallel to the NW axis, the sin $\alpha$ term in eq 2 can be neglected. This leads to the following two (angle averaged) expressions for the maximum and minimum photocurrent within the NW network

$$
I_{\max }(\beta=0) \propto E_{0}^{2} E_{\mathrm{dc}}^{2}\left(\frac{2}{3}\left|\frac{2 \varepsilon_{\mathrm{m}}}{\varepsilon+\varepsilon_{\mathrm{m}}}\right|^{2}+\frac{4}{3}\right)
$$

and

$$
I_{\min }(\beta=90) \propto E_{0}^{2} E_{\mathrm{dc}}^{2}\left(\frac{4}{3}\left|\frac{2 \varepsilon_{\mathrm{m}}}{\varepsilon+\varepsilon_{\mathrm{m}}}\right|^{2}+\frac{2}{3}\right)
$$

The resulting polarization anisotropy is then 


$$
\rho=\frac{I_{\max }-I_{\min }}{I_{\max }+I_{\min }}=\frac{1}{3}\left(1-\left|\frac{2 \varepsilon_{\mathrm{m}}}{\varepsilon+\varepsilon_{\mathrm{m}}}\right|^{2}\right) /\left(1+\left|\frac{2 \varepsilon_{\mathrm{m}}}{\varepsilon+\varepsilon_{\mathrm{m}}}\right|^{2}\right)
$$

and predicts anisotropy values of $\rho_{\mathrm{CdSe}}=0.258\left(\rho_{\mathrm{CdTe}}=\right.$ 0.272) for CdSe (CdTe) NWs. The remarkable agreement between these model $\rho$-values and actual experimental anisotropies [CdSe: $\rho=0.25(\sigma=0.04)$; CdTe: $\rho=0.22$ $(\sigma=0.04)]$ is likely a coincidence given the rudimentary nature of the model. However, the model's prediction of nonzero anisotropies again supports the existence of residual polarization sensitivities in random NW networks. Full expressions for $I_{\max }, I_{\min }$, and $\rho$, all including the sin $\alpha$ term, can be found in the Supporting Information.

Additional corroborating evidence include experiments aimed at examining the magnitude of the observed photocurrent as a function of applied bias. Specifically, eq 5 implies that the photocurrent scales as $E_{\mathrm{dc}}^{2}$ (or as $V^{2}$ ). Results from these experiments are shown in Figure $4 \mathrm{~b}$ and suggestively indicate a linear dependence between $I$ and $V^{2}$. It should be noted, however, that this behavior can also be explained by space-charge limited current flow within the nanowire network. ${ }^{31}$ As a consequence, more detailed experiment and modeling are required in order to more unambiguously describe carrier transport in the random NW network.

Conclusion. This study has demonstrated the existence of sizable polarization anisotropies in photodetectors made of random NW networks. Anisotropy values of $\rho=0.25$ ( $\sigma$ $=0.04)[\rho=0.22(\sigma=0.04)]$ were measured and are nearly identical in magnitude to those observed from ensembles of dielectrophoretically aligned NWs. The underlying reason for the existence of such anisotropies is thought to be 2-fold: (a) the directionality imposed by the external dc field when dissociating strongly bound 1D excitons within NWs aligned near parallel to $\vec{E}_{\mathrm{dc}}$ and (b) the selective excitation of such wires with linearly polarized light when $\vec{E}_{0} \mid \vec{E}_{\mathrm{dc}}$. As a consequence, a strong underlying asymmetry exists in the device even though the random NW network as a whole exhibits no preferred orientation. A simple geometric model has been developed to qualitatively explain the data. Both model and experiment, in turn, suggest the feasibility of realizing polarization sensitive NW photodetectors without the need to align NWs.

Acknowledgment. We thank Chagaan Bataar of the Office of Naval Research for financial support. M. K. also thanks the NSF CAREER program (CHE-0547784), the NSF NER program (ECS-0609172), the Research Corporation and the Notre Dame Radiation Laboratory/Department of Energy, Office of Basic Energy Sciences for financial support and for use of their facilities. Masaru Kuno is a Cottrell Scholar of Research Corporation. D. J. thanks the NSF CAREER program (DMR-0645698) for financial support.

Supporting Information Available: Additional low and high resolution TEM images of CdTe NWs. Cartoon schematic illustrating the device fabrication. $I-V$ measurements on a $\mathrm{CdSe}$ random $\mathrm{NW}$ network. Photocurrent anisotropy measurement from a random CdTe NW network. CdTe photocurrent action spectrum and corresponding UV/ visible extinction spectrum. Spot size independence of the polarization anisotropy. Phase angle insensitivity of the polarization anisotropy across the gap. Frequency and spectral bandwidth measurements of the polarization anisotropy. Full model expressions for $I_{\max }, I_{\min }$, and $\rho$. This material is available free of charge via the Internet at http:// pubs.acs.org.

\section{References}

(1) Wang, J. F.; Gudiksen, M. S.; Duan, X. F.; Cui, Y.; Lieber, C. M. Science 2001, 293, 1455-1457.

(2) Huang, M. H.; Mao, S.; Feick, H.; Yan, H. Q.; Wu, Y. Y.; Kind, H.; Weber, E.; Russo, R.; Yang, P. D. Science 2001, 292, 1897-1899.

(3) Mao, S. S. Int. J. Nanotechnol. 2004, 1, 42-85.

(4) Gradecak, S.; Qian, F.; Li, Y.; Park, H. G.; Lieber, C. M. Appl. Phys. Lett. 2005, 87, 173111-1-173111-3.

(5) Sakaki, H. Jpn. J. Appl. Phys. 1980, 19, L735-L738.

(6) Lu, W.; Xiang, J.; Timko, B. P.; Wu, Y.; Lieber, C. M. Proc. Natl. Acad. Sci. U.S.A. 2005, 102, 10046-10051.

(7) Zhou, X.; Dayeh, S. A.; Aplin, D.; Wang, D.; Yu, E. T. Appl. Phys. Lett. 2006, 89, 053113.

(8) Vouilloz, F.; Oberli, D. Y.; Dupertuis, M. A.; Gustafsson, A.; Reinhardt, F.; Kapon, E. Phys. Rev. B 1998, 57, 12378.

(9) Someya, T.; Akiyama, H.; Sakaki, H. Phys. Rev. Lett. 1995, 74, 36643667.

(10) Ils, P.; Gréus, Ch.; Forchel, A.; Kulakovskii, V. D.; Gippius, N. A.; Tikhodeev, S. G. Phys. Rev. B 1995, 51, 4272.

(11) Zhou, R.; Chang, H. C.; Protasenko, V.; Kuno, M.; Singh, A. K.; Jena, D.; Xing, H. J. Appl. Phys. 2007, 101, 073704-1-073704-9.

(12) Ruda, H. E.; Shik, A. J. Appl. Phys. 2006, 100, 024314-1-024314-5.

(13) Sercel, P. C.; Vahala, K. J. Phys. Rev. B 1991, 44, 5681.

(14) McIntyre, C. R.; Sham, L. J. Phys. Rev. B 1992, 45, 9443-9446.

(15) Ruda, H. E.; Shik, A. Phys. Rev. B 2005, 72, 115308-1-115308-11.

(16) Singh, A.; Li, X.; Protasenko, V.; Galantai, G.; Kuno, M.; Xing, H.; Jena, D. Nano Lett. 2007, 7, 2999.

(17) Kravtsova, Y.; Krull, U.; Musikhin, S. F.; Levina, L.; Ruda, H. E.; Shik, A. Appl. Phys. Lett. 2007, 90, 083120-1-083120-3.

(18) Gudiksen, M. S.; Wang, J.; Lieber, C. M. J. Phys. Chem. B 2002, 106, 4036.

(19) Grebinski, J. W.; Richter, K. L.; Zhang, J.; Kosel, T. H.; Kuno, M. J. Phys. Chem. B 2004, 108, 9745-9751.

(20) Grebinski, J. W.; Hull, K. L.; Zhang, J.; Kosel, T. H.; Kuno, M. Chem. Mater. 2004, 16, 5260-5272.

(21) Kuno, M.; Ahmad, O.; Protasenko, V.; Bacinello, D.; Kosel, T. H. Chem. Mater. 2006, 18, 5722-5732.

(22) Landau, L. D.; Lifshits, E. M. Electrodynamics of Continuous Media; Pergamon: New York, 1984; 34-41..

(23) (a) Palik, E. D. Handbook of Optical Constants of Solids; Academic Press: New York, 1985; 409-427. (b) Palik, E. D. Handbook of Optical Constants of Solids II: Academic Press: New York, 1998; 579.

(24) Protasenko, V.; Bacinello, D.; Kuno, M. J. Phys. Chem. B 2006, 110, 25322-25331.

(25) Dneprovskii, V. S.; Zhukov, E. A.; Shalygina, O. A.; Lyaskovskii, V. L.; Muljarov, E. A.; Gavrilov, S. A.; Masumoto, Y. J. Exp. Theor. Phys. 2002, 94, 1169-1175.

(26) (a) Muljarov, E. A.; Zhukov, E. A.; Dneprovskii, V. S.; Masumoto, Y. Phys.Rev. B 2000, 62, 7420-7432. (b) Shabaev, A.; Efros, Al. L. Nano Lett. 2004, 4, 1821.

(27) Perebeinos, V.; Tersoff, J.; Avouris, P. Phys. Rev. Lett. 2004, 92, 257402-1.

(28) Robel, I.; Bunker, B. A.; Kamat, P. V.; Kuno, M. Nano Lett. 2006, 6, 1344.

(29) Kumar, S.; Murthy, J. Y.; Alam, M. A. Phys. Rev. Lett. 2005, 95, 066802-1-066802-3.

(30) Huang, Y.; Duan, X. F.; Cui, Y.; Lieber, C. M. Nano. Lett. 2002, 2 , 101-104.

(31) Lampert, M. A. Phys. Rev. 1956, 103, 1648.

NL080028P 